\title{
Optimization of heat-dried silane application for CAD/CAM ceramic resin bonding
} MAGNE P $^{1}$, BRUZI G $^{2}$, CARVALHO $^{3}$, ENCISO R $^{4}$, GIANNINI ${ }^{5}$

${ }^{1}$ Don and Sybil Harrington Professor of Esthetic Dentistry, Restorative Sciences, Ostrow School of Dentistry, University of Southern California, Los Angeles, California, USA.

${ }^{2}$ Departament of Operative Dentistry, Operative Dentistry, Federal University of Alfenas, Alfenas, Minas Gerais, Brazil.

${ }^{3}$ Departament of Health , Integrated Clinic, State University of Southwest Bahia, Feira de Santana, Bahia, Brazil. aoc1981@hotmail.com

${ }^{4}$ Assistant Professor of Clinical Dentistry Division of Endodontics, Oral and Maxillofacial Surgery and Orthodontics, Ostrow School of Dentistry, University of Southern California, Los Angeles, California, USA.

${ }^{5}$ Associate Professor, Department of Restorative Dentistry, Piracicaba School of Dentistry, Campinas State University, Piracicaba, São Paulo, Brazil. Abstract:Aim:Evaluate surface treatments and silane application methods and their effect on shear bond strength (SBS) before and after aging. Materials and methods. Slices of IPS e.max CAD and Vitablocs Mark II, were embedded in acrylic resin. The block was randomly assigned to 5 groups; E:etching with hydrofluoric acid (HF), rinsing, followed by cleaning in ultrasonic bath; E/S:HFetching and cleaned as group E followed by silane application for 20s, air drying and hot drying; E/S+:HFetching and cleaned as group E, followed by silane application for 60s, air drying and hot air drying, rinsing with boiling water and hot air drying; S:silane application for 20s followed by air drying and hot air drying; $\mathrm{S}+$ :silane application for 60 s followed by air drying, air drying and hot air drying, rinsing in boiling water and hot drying. Cylinders of composite resin $(n=12)$ were bonded with adhesive. SBS testing was carried out after $24 \mathrm{~h}$ or after thermocycling for groups E; E/S and E/S+. Results. For 24h SBS showed sign

Keywords: adhesion CAD/CAM; ceramic; silane.

\section{Introduction}

Bonding to tooth hard tissues enables minimally invasive restorative techniques that do not require mechanical retention principles. This "biomimetic approach" is valid for direct, semi-direct and indirect procedures. In the case of silicabased ceramic inlays/onlays/veneers, a reliable resin bond depends mainly on two mechanisms. First, the hydrofluoricacid etching (HF-etching) is a mechanical interlocking through microretentions in the ceramic bonding surface. Second, the chemical union promoted by a silane coupling agent allows covalent bonding between two different substrates (organic and inorganic). ${ }^{[20,27,16]}$

Despite the obvious function of each of these steps, the real necessity of combining micro-mechanical and chemical bonding have resulted in different conclusions. While some authors revealed that an appropriate silane application is sufficient to obtain a durable resin-ceramic bonding ${ }^{[18,12,10]}$ others claim that HF-etching is required. ${ }^{[45,43,38,44]}$ In fact, there are some valid justifications trying to eliminate the HF-etching step. First HF acid can

cause potential injury to health, when it is not properly handled, due to its high toxicity. ${ }^{[24,31]}$ Second, HFetching generates insoluble silica-fluoride salts at the surface of the cermic. Those can negatively influence the resin-ceramic bond strength unless they are removed by ultrasonic cleaning. ${ }^{[23,1]}$

Eliminating the HF-etching step and relying essentially on chemical coupling implies that the most stable and durable siloxane bond be obtained. Hydrolytic degradation can affect the stability of the chemical bond. It has been 
clearly demonstrated that silane efficiency can be enhanced by heat drying $\left(50^{\circ} \mathrm{C}\right.$ to $\left.100^{\circ} \mathrm{C}\right)$, which eliminates water, solvents and by-products allowing covalent bond, promoting a completed condensation reaction and crosslinking of the silane film. ${ }^{[10,42,25,32,37]} \mathrm{A}$ further step in the optimization of silane application has been proposed by Hooshmand $e t$ $a l .(2002)^{[18]}$ in order to isolate the covalently bound silane monolayer at the ceramic surface. In practice, when a silane is applied to a substrate, multiple layers are forming: (1) the outermost coat, that present small oligomers, which consist in weak oligomeric siloxanols, can be removed by organic solvents or water; (2) the middle film consists in a comparable coat as the first one but also presents some siloxane bonds (covalent) between the silane molecules (loosely bound oligomers); this coat can be removed by hot water and finally, (3) the inner coat is the chemically reacted region, the silane monolayer itself, which is more stable and contributes to the bond strength. ${ }^{[41]}$ The optimized silane application aims at the elimination of all loosely bound oligomers by hot water rinsing and hot air drying.

There is a lack of data about the aging of resin-ceramic bonds generated from this optimized silane application method and whether there is a substantial benefit to justify the additional steps. Another concern is the omission HF-etching proposed by some authors as a consequence of the optimized silane application. Hence, in this study, the effect of various combinations of surface treatments, silane application methods and their effect on the resin-ceramic shear bond strength (SBS) before and after aging were investigated. The first null hypothesis was that there is equal contribution of mechanical interlocking and chemical coupling to the resin-ceramic bond and that the optimized silane coating will not differ from the other groups. Second, simulated aging will not have an influence in the SBS for the various treatments. Third, the ceramic material (feldspathic porcelain vs. lithium disilicate) will not affect the performance of the resin-ceramic bond.

\section{Materials and Methods}

Sixteen 2mm-thick slices $(14 \times 16 \mathrm{~mm})$ of IPS e.max CAD (EMAX; Ivoclar Vivadent, AG, Schann, Liechtenstein) and Vitablocs Mark II (VMII; VITA Zahnfabrik GmbH, Bad Säckingen, Germany) were obtained by sectioning CAD/CAM blocks with a slow-speed diamond water-cooled saw (Isomet, Buehler, Lake Bluff, IL, USA). The EMAX slices were crystalized in a ceramic furnace (Austromat 624; DEKEMA Dental-Keramiköfen GmgH, Freilassing, Germany) following the manufacturer's instructions. All the slices were embedded in an acrylic resin positioning base (Palapress; Heraeus Kulzer, Hanau, Germany) and polished with 400- and 600-grit silicon carbide paper under water cooling. The surfaces of all slices were then cleaned in an ultrasonic bath in distilled water during 5 min. Five surface conditioning methods were applied: E - 5\% HF-etching (IPS Ceramic Etching Gel, Ivoclar Vivadent) for 20s for EMAX and 60s for VMII, rinsed for 60s with air/water spray followed by post-etching cleaning in ultrasonic bath in distilled water for $2 \mathrm{~min}$; E/S - 5\% HF-etching as above, silane (Silane, Ultradent, South Jordan, Utah) applied for 20s and air dried for $20 \mathrm{~s}$, hot dried at $60^{\circ} \mathrm{C}$ for $20 \mathrm{~s}$ (with hair dryer at $10 \mathrm{~cm}$-distance); E/S+ - 5\% HF-etching as above, silane applied for $60 \mathrm{~s}$, air dried for $20 \mathrm{~s}$ and hot air dried $60^{\circ} \mathrm{C}$ for another $20 \mathrm{~s}$ rinsed with boiling water for $15 \mathrm{~s}$ and hot air dried again for $20 \mathrm{~s} ; \mathrm{S}-$ no etching, silane for $20 \mathrm{~s}$ and air dried for $20 \mathrm{~s}$, hot dried at $60^{\circ} \mathrm{C}$ for $20 \mathrm{~s}$ or S + - no etching, silane applied for $60 \mathrm{~s}$, air dried for $20 \mathrm{~s}$ and hot air dried $60^{\circ} \mathrm{C}$ for another $20 \mathrm{~s}$ (with hair dryer at $10 \mathrm{~cm}$-distance), rinsed with boiling water for $15 \mathrm{~s}$ and hot air dried again for $20 \mathrm{~s}$.

Specimens were then placed in a positioning device and a teflon mold (Ultradent Jig) containing a bonding area $0.04448 \mathrm{~cm} 2$ was used to build composite resin cylinders. First, the adhesive system (Optibond FL, Kerr Corp, Orange, California, USA) was applied to the ceramic, then the restorative composite resin Z100 (3M-ESPE) was inserted into the mold in two increments of approximately $1.8 \mathrm{~mm}$ each using a small condensing spatula. Each increment was light polymerized for 20s (Valo, Ultradent) at $1000 \mathrm{~mW} / \mathrm{cm}^{2}$. Six resin cylinders (diameter: $2.4 \mathrm{~mm}$ ) were built onto each ceramic slice (length: $15 \mathrm{~mm}$, width: $4 \mathrm{~mm}$, thickness: $2 \mathrm{~mm}$ ), $3.5 \mathrm{~mm}$ apart, resulting in 12 specimens per group. The test was performed after $24 \mathrm{~h}$ of storage in distilled water. For groups $\mathrm{E} ; \mathrm{E} / \mathrm{S}$ and $\mathrm{E} / \mathrm{S}+$ additional samples (12/group) were built and subjected to simulated aging by thermocycling for 20,000 cycles at temperatures alternating between $5^{\circ} \mathrm{C}$ and $55^{\circ} \mathrm{C}$ with an dwell time of 30s (Thermocycler, SD Mechatronik Gmbh, Feldkirchen-Westerham, Germany) with a 
transfer time between baths of 15s. All samples were submitted to SBS testing (Shear Bond Tester; Bisco Inc., Schaumburg, IL) with a ramp load of $43.8 \mathrm{~kg}$-force per minute. The failure mode was assessed by macro-photography (Nikon D60 and Sigma Lens $105 \mathrm{~mm}$ and a teleconverter x2).

\section{Ethics Statement}

The mean bond strength and standard deviation were calculated for all groups. Statistical analysis was performed using multifactorial analyses of variance (ANOVA), in which surface conditioning method; ceramic substrate and aging served as independent factor. The Tukey HSD test was used for equal variances to test the average values of all groups. The significance level adopted in all analyses was $\leq 0.05$ ( $<<0.05$ ) (MedCalc Software for Windows version 12.3.0, Mariakerke,

Belgium).

\section{Results}

According to the two-way ANOVA (used for comparison of the baseline values of the five surface conditioning treatments before aging), both the ceramic substrate and the surface conditioning method had an influence on the $24 \mathrm{~h}$ SBS values. Tukey HSD test $(p<0.05)$ showed that $E / S$ had significantly higher bond strength $(p<0.005)$, for both ceramic (EMAX 21,06MPa; VMII 24,27MPa), compared to E and E/S+ (range: $15.50 \mathrm{MPa}$ to 20.96). The silane application alone ( $\mathrm{S}$ and $\mathrm{S}+$ ) resulted in significantly weaker bond strength, independent of the ceramic (EMAX 3,92 to 4,32MPa; VMII 5,69 to 5,91MPa). There were 5 specimens for group $\mathrm{S}$ and 3 for group $\mathrm{S}+$ showing pre-test failure, which were considered as $0 \mathrm{MPa}$ in the statistical analyses. No significant differences were found between $\mathrm{E} / \mathrm{S}$ and $\mathrm{E} / \mathrm{S}+$ for VMII $(\mathrm{p}>0.05)$.

Groups $\mathrm{S}$ and $\mathrm{S}+$ were not subjected to the aging step because of their poor baseline values and pretest failures. Three-way ANOVA (ceramic substrate, surface conditioning method and aging as factors) confirmed the significant effect of the substrate and the surface conditioning method. Tukey HSD tests revealed that VMII remained superior to EMAX after aging $(\mathrm{p}<.001)$. For surface conditioning, aging did not seem to have an effect on group E (22MPa vs. $21 \mathrm{MPa}$ at baseline for VITA and $14 \mathrm{MPa}$ vs. $15 \mathrm{MPa}$ at baseline for IPS e.max) $(\mathrm{p}=.17)$. There was a decrease of $22-29 \%$ of SBS values due to aging in group E/S (19MPa vs. 24MPa at baseline for VITA and 16MPa vs. 21MPa at baseline for IPS e.max) ( $\mathrm{p}=.004)$. Aging had an opposite effect in $\mathrm{E} / \mathrm{S}+$ with a $17-31 \%$ increase of SBS values (25MPa vs. 19MPaat baseline for VITA and $21 \mathrm{MPa}$ vs. $15 \mathrm{MPa}$ at baseline) $(\mathrm{p}=.034)$. Graphic 1 presents the means and standard deviations SBS values for each group, before and after thermocycling.

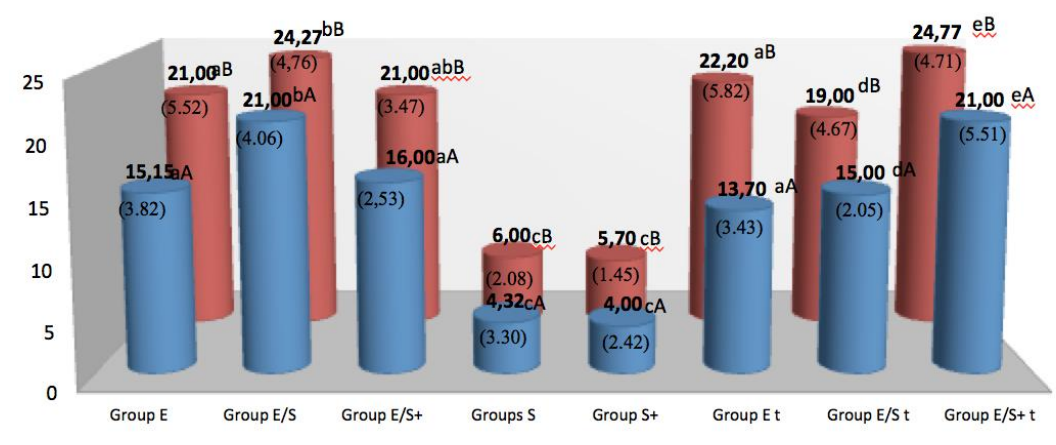

$\square$ EMAX $\square$ VITA

Figure 1; While the failure mode for IPS e.max CAD was always adhesive, Vitablocs Mark II failed cohesively when etched (unless aged in $\mathrm{E}$ and $\mathrm{E} / \mathrm{S})$. 


\begin{tabular}{|c|c|c|c|c|}
\hline \multirow[t]{3}{*}{ Silane application } & \multicolumn{4}{|c|}{ Tvpe of failure } \\
\hline & \multicolumn{2}{|c|}{ EMAX } & \multicolumn{2}{|c|}{ VITA } \\
\hline & Adhesive & Cohesive & Adhesive & Cohesive \\
\hline $\mathrm{E}$ & 100 & 0 & 0 & 100 \\
\hline $\mathrm{E} / \mathrm{S}$ & 100 & 0 & 0 & 100 \\
\hline $\mathrm{E} / \mathrm{S}+$ & 100 & 0 & 0 & 100 \\
\hline $\mathrm{S}$ & 100 & 0 & 100 & 0 \\
\hline $\mathrm{S}$ & 100 & 0 & 100 & 0 \\
\hline E thermocycling & 100 & 0 & 25 & 75 \\
\hline $\mathrm{E} / \mathrm{S}$ thermocycling & 100 & 0 & 75 & 25 \\
\hline $\mathrm{E} / \mathrm{S}+$ thermocycling & 100 & 0 & 0 & 100 \\
\hline
\end{tabular}

Table 1. Presents the failure mode for each group, before and after thermocycling.

\section{Discussion}

The microtensile bond strength (MTBS) test is usually preferred to evaluate interfacial adhesives because it generates uniform stress distribution across the bonded interface, ${ }^{[33]}$ limiting the possibility of cohesive failure in the substrate. ${ }^{[41]}$ Several other methods (shear, micro-shear, and tensile) can be used to determine in vitro the bond strength between resin luting agents and ceramics. None of these tests are accepted as a universal method and each of them have their own advantages and limitations. Several aspects must be taken into consideration when selecting a test method, such as the technique sensitivity (e.g. MTBS can present a high frequency of premature failures, ${ }^{[34]}$ and it can be affected by cutting speed, ${ }^{[39]}$ shape of the sample and the brittleness of the substrate $)^{[15,11]}$ and the magnitude of the bond strength being investigated (strong bonds tend to generate more cohesive failures, which calls for specific tests). From a practical standpoint, the SBS test is considered a low technique-sensitive test as it avoids the specimen sectioning and trimming steps that can introduce early micro-cracking of fragile substrates. ${ }^{[15,11,19]}$ As a result, some authors concluded that a shear approach was more appropriate compared to a microtensile approach., ${ }^{2,14}$ Based on the above description, the main reason for choosing SBS as a test method was to avoid damage and generate cracks and defects that could influence the stress distribution in the bond interface. While it proved to be appropriate when using EMAX as a substrate (100\% of adhesive failures), the SBS test generated a high percentage of cohesive failures with VMII when HF etching was used. This would normally mean that the substrate itself was evaluated rather than the bond. However, when looking at the results in figure 1, it is clear that the same trend is observed for both EMAX and VMII. There is a clear evidence that the use of the silane alone resulted in similar consequences for both materials (low SBS of 4-6MPa and $100 \%$ adhesive failures), which is in support of the present methods and data.

The adhesive performance in the course of time can be evaluated by thermocycling, the role of which is not necessarily to reproduce thermal stresses in the oral environment. Due to coefficient of thermal expansion differences between resins and ceramics, thermocycling also induces a mechanical fatigue phenomenon and hydrolytic degradation that accelerate the aging process resulting from many other factors in vivo. The number of cycles, temperatures and dwell time differ considerably between reports (300 to $1,000.000$ cycles; temperatures of 0 to $67^{\circ} \mathrm{C}$; dwell time of $15 \mathrm{~s}$ to $120 \mathrm{~s}$ ). According to Gale and Darvell (1999 review) ${ }^{[14]}, 10,000$ cycles correlate with approximately one year of clinical service. The thermocycling protocol proposed in this study was 20,000 cycles and the primary goal was the simulation of thermally-induced mechanical loading. Only groups E, E/S, and E/S+ were subjected to thermocycling based on their excellent SBS values after 24h storage in distilled water. The use of silane alone (S and S+) not only resulted in poor baseline values at $24 \mathrm{~h}$ but also featured the only pre-failure test samples. Therefore it was decided not to proceed with thermocycling with those "silane alone" groups. 
The simulation of aging can affect significantly the SBS values in different ways. For group E, independent of the ceramic substrate, the values were not statistically different before and after aging, which is in agreement with Roulet, Söderholm, Longmate (1995), ${ }^{[38]}$ who found no difference in resin-ceramic SBS after 12 months of storage in water. This reaffirms the importance of surface roughness, which is able to increase contact area, wettability and surface energy of the ceramic substrate. ${ }^{[7,30]}$ It also creates mechanical interlocking for the luting agent. Another factor that should be considered is the composite resin degradation, which is material dependent. ${ }^{[21,35]}$ The luting composite plays an important role in the final resin-ceramic bond strength.35 In the present study the wetting resin (Optibond FL, bottle no. 2) is $48 \%$ filled and was associated with a highly-filled restorative composite resin. This can explain the high resistance to thermally-induced mechanical fatigue and hydrolysis even after 20,000 cycles. The use of a restorative composite as a luting composite is recommended because of the improved physic-mechanical properties, color stability and working time when compared to dual-cure or flowable composite resins. ${ }^{[5]}$ Film thickness, which is normally limitation with restorative composites resins, can be resolved using preheating. ${ }^{[3-6,13,22,26,46,48]}$

When using EMAX, group E presented a stable SBS values after aging but was still significantly inferior to that of E/S+. This emphasizes the synergetic effect of etching and silane on that specific ceramic. Those differences were much less obvious for VMII possibly because of the cohesive failure mode that may have had a "masking effect" on the data (in this case the bond strength often exceeded the cohesive strength of the ceramic). Both EMAX and VMII E/S groups had significantly lower SBS values after thermocycling (15MPa and $19 \mathrm{MPa}$, respectively). The constant contact with water can induce hydrolysis of the siloxane bond (-Si-O-Si) and accelerate the degradation of the silane coupling agent. ${ }^{28}$ The amount of hydrolytic damage is related to the proportion of siloxane bonds within the condensed layers of silane coupling agent and the amount of siloxane bonds between the monolayer and the ceramic surface. Because the outmost condensed layers in E/S were not rinsed, those instable chains with small oligomers could explain the weaker bond between ceramic and composite resin over aging. These findings are in contrast with some previous studies were the aged groups did not differ from the non-aged group in bond strength. ${ }^{[17]}$ The explanation for this difference may lie in the aging period, varying between $1 \mathrm{~h}$ and 5,000 cycles vs. 20,000 cycles in the present work.

For either ceramics, the best resin SBS after aging was obtained through the E/S+ treatment. This optimal silane application, as defined by Hooshmand et al. ${ }^{[18]}$ aims at saturating the substrate surface with silane (relatively long application time of $60 \mathrm{~s}$ ), securing the siloxane bond of the monolayer (two consecutive heat treatment, one before and one after hot rinsing) and eliminating the outmost loosely bond silane layers (hot rinsing). It appears from the results that this optimal application might not have had a tangible effect immediately or at $24 \mathrm{~h}$. However, the post-aging results speak in favor of that technique. In the E/S treatment, the silane was applied only for $20 \mathrm{~s}$ in order to prevent the formation of the loosely bound oligomer layers but this might not have been sufficient to create a dense monolayer able to withstand the aging process. Rinsing with boiling water in the optimal application technique (E/S+) requires special care to avoid thermal shock to thin ceramic restorations such as porcelain veneers. If no internal residual stresses (from the firing process) are present, the ceramic should not suffer from the thermal stress. HF etching, however, significantly weakens the ceramic ${ }^{8}$ and further research should explore the crack propensity of thin etched porcelain subjected to thermal stresses during the rinsing of the silane, even though no cracks were observed in the 2-mm thick specimens of this study. Another parameter to explore would be the difference between CAD/CAM and hand layered ceramics, which may have different residual stresses from firing and therefore different risk of thermally-induced cracking.

An hypothesis can be the fact that the silane condensation reaction continues with time and can be more resistant to the effect of long time water exposure, proving the stability of the inner silane layer.

In this study 5\% HF-etching was applied during 20s for EMAX and 60s for VMII. This protocol was based on previous studies showing that overetching can impair the ceramic substrate by producing microporosities and microcracks. ${ }^{[38,47]}$ To confirm this finding a pilot test accomplished by the present authors using EMAX to compare two $\mathrm{HF}$ concentrations, $10 \% \mathrm{HF}$ and $5 \% \mathrm{HF}$ followed by silane application and hot air drying. The results showed significant difference in SBS values: $17 \mathrm{MPa}$ with $10 \% \mathrm{HF}$ vs. $21 \mathrm{MPa}$ with $5 \% \mathrm{HF}(\mathrm{p}<.01)$. For the clinician, this 
definitely stresses out the importance of knowing not only the generic name of a product but also the details about composition and concentration. HF acid is more commonly used as $10 \%$ for the porcelain veneer technique, ${ }^{[23]}$ therefore practitioners would be tempted to use the same product for all types of ceramics.

Based on the results of the present study, the original works of Stangel et al. (1987) ${ }^{[45]}$ still hold true and it cannot be recommended to omit HF-etching from the conditioning steps. Claims about HF potential injury to health when not properly handled are limited today because of the gel forms that limit the production of fumes and allow precise placement without splashing. However, HF generates insoluble silica-fluoride salts that can influence the resin-ceramic bond strength and must be removed by post-etching ultrasonic cleaning. ${ }^{[23,1]}$ Failed attempts to eliminate the HF-etching step (Groups S and $\mathrm{S}+$ ) confirm the fundamental contribution of the micromechanical bond. Those results may seem to be in contrast with Hooshmand et al. (2002), ${ }^{[18]}$ who found that the optimal silane application was capable to provide a durable resin-ceramic tensile bond. However, the effect of HF-etching associated with a silane was not evaluated and HF-etching was not following by post-etching cleaning. Fabianelli et al. $(2010)^{[10]}$ were able to obtain similar bond strength to IPS Empress when combining HF-etching/ heat-dried silane or when using the heat-dried silane alone. However, it is know from Della Bona and Van Noort (1998) ${ }^{[36]}$ that IPS Empress, unlike IPS e.max, is not a favorable substrate for HF etching. In addition, no postetching cleaning and no aging process were carried out by Fabianelli et al. (2010). ${ }^{[10]}$ Omission of simulated aging probably offer figures that misrepresent the actual contribution of silane coupling agents to the long-term in vivo bond strengths. ${ }^{[36]}$

The failure mode, analyzed by macrophotography, showed $100 \%$ of adhesive failure with EMAX (all groups). This accounts for the high cohesive strength of the lithium disilicate substrate. On the other hand, VMII displayed 100\% adhesive failure only in groups where the HF-etching was omitted ( $\mathrm{S}$ and $\mathrm{S}+$ ). When HF-etching was applied to VMII and aging was simulated adhesive failures were still found except for E/S+, the only group in this study with $100 \%$ cohesive failure after aging. Even though this represents a weakness in the experimental method, it still speaks in favor of the optimal silane application in combination to HF etching.

The results from this study are limited to an in vitro setup and only one silane coupling agent. Knowing that ceramic primers including not only silane but also other monomers such as MDP are available on the market and can vary from one another, further research should focus on those materials, as well as other practical methods for the hot rinsing step such as the use of steam cleaning.

\section{Conclusion}

From this in-vitro study, it can be concluded that the silane application method and the simulated aging affected significantly the SBS values for both feldpathic porcelain and lithium disilicate ceramic. The combination of HF etching (including post-etching cleaning) and heat-dried silane coupling proved superior to the use of a single conditioning method. HF-etching as a single treatment proved superior compared to the heat-dried silane coupling alone. Furthermore, the results showed that the optimal silane application (hot water rinsing) was significant in maintaining a high resin ceramic CAD/CAM SBS values after simulated aging (20,000 cycles equivalent to 2 years of service).

Author Contributions (as necessary)

MAGNE P - Contribution to the paper: Idea, wrote the paper.

BRUZI G - Contribution to the paper: Wrote the paper, performed the experiments in partial fulfillment of requirements for a degree.

CARVALHO AO - Contribution to the paper: Wrote the paper, performed the experiments in partial fulfillment of requirements for a degree.

ENCISO R - Contribution to the paper: performed statistical evaluation.

GIANNINI M - Contribution to the paper: Wrote the paper.

Acknowledgements (as necessary)

The authors wish to express their gratitude to Ivoclar Vivadent for providing IPS e.max CAD and IPS Ceramic 
Etching Gel; Kerr Crop. for providing Optibond FL Adhesive and Ultradent for providing Silane. Study supported in part by CAPES Foundation 4979/11-7 (Dr. Bruzi) and CNPq 20092/2011-6 (Dr. Carvalho). The authors would like to express their gratitude to LCME-UFSC and UFLA's lab, with all the help in the SEM images.

\section{References}

1. Belli R, Guimarães JC, Filho AM, et al. Post-etching cleaning and resin/ceramic bonding: Microtensile bond strength and EDX analysis. J Adhes Dent 2010; 12: 295-303.

2. Beloica M, Goracci C, Carvalho CA, et al. Microtensile vs microshear bond strength of all-in-one adhesives to unground enamel. J Adhes Dent 2010; 12: 427-33.

3. Besek M, Mörmann WH, Persi C, et al. The curing of composites under Cerec inlays. Schweiz Monatsschr Zahnmed 1995; 105: 1123-8. Abstract.

4. Daronch M, Rueggeberg FA, De Goes MF, et al. Polymerization kinetics of pre-heated composite. J Dent Res 2006; 85: 38-43.

5. Daronch M, Rueggeberg FA, De Goes MF. Monomer conversion of pre-heated composite. J Dent Res 2005; 84: 663-7.

6. Deb S, Di Silvio L, Mackler HE, et al. Pre-warming of dental composites. Dent Mater 2011; 27: e51-9.

7. Della Bona A, Shen C, Anusavice KJ. Work of adhesion of resin on treated lithia disilicate-based ceramic. Dent Mater 2004; 20: 338-44.

8. Della Bona A, van Noort R. Ceramic surface preparations for resin bonding. Am J Dent 1998; 11: 276-80.

9. El Zohairy AA, Saber MH, Abdalla AI, et al. Efficacy of microtensile versus microshear bond testing for evaluation of bond strength of dental adhesive systems to enamel. Dent Mater. 2010; 26(9): 848-54.

10. Fabianelli A, Pollington S, Papacchini F, et al. The effect of different surface treatments on bond strength between leucite reinforced feldspathic ceramic and composite resin. J Dent 2010; 38: 39-43.

11. Ferrari M, Goracci C, Sadek F, et al. Microtensile bond strength tests: scanning electron microscopy evaluation of sample integrity before testing. Eur J Oral Sci 2002; 110: 385-91.

12. Filho AM, Vieira LC, Araújo E, et al. Effect of different ceramic surface treatments on resin microtensile bond strength. J Prosthodont 2004; 13: 28-35.

13. Fróes-Salgado NR, Silva LM, Kawano Y, et al. Composite pre-heating: effects on marginal adaptation, degree of conversion and mechanical properties. Dent Mater 2010; 26: 908-14.

14. Gale MS, Darvell BW. Thermal cycling procedures for laboratory testing of dental restorations. J Dent 1999; 27: 89-99.

15. Goracci C, Sadek FT, Monticelli F, et al. Influence of substrate, shape, and thickness on microtensile specimens' structural integrity and their measured bond strengths. Dent Mater 2004; 20: 643-54.

16. Ho GW, Matinlinna JP. Evaluation of the microtensile bond strength between resin composite and hydrofluoric acid etched ceramic in different storage media. J Adhes Sci Technol 2011; 25: 2671-85.

17. Hooshmand T, Parvizi S, Keshvad A. Effect of surface acid etching on the biaxial flexural strength of two hot-pressed glass ceramics. J Prosthodont 2008; 17: 415-9.

18. Hooshmand T, van Noort R, Keshvad A. Bond durability of the resin-bonded and silane treated ceramic surface. Dent Mater 2002; 182: 179-88.

19. Ishikawa A, Shimada Y, Foxton RM, et al. Microtensile and micro-shear bond strengths of current self-etch adhesives to enamel and dentin. Am J Dent 2007; 20: 161-6. (abstrat)

20. Jardel V, Degrange M, Picard B, et al. Surface energy of etched ceramic. Int J Prosthodont 1999; 12: 415-8.

21. Liu Q, Meng X, Yoshida K, et al. Bond degradation behavior of self-adhesive cement and conventional resin cements bonded to silanized ceramic. J Prosthet Dent 2011; 105: 177-84.

22. Lucey S, Lynch CD, Ray NJ, et al. Effect of pre-heating on the viscosity and microhardness of a resin composite. J Oral Rehabil 2010; 37: 278-82.

23. Magne P, Cascione D. Influence of post-etching cleaning and connecting porcelain on the microtensile bond strength of composite resin to feldspathic porcelain. J Prosthet Dent 2006; 96: 354-61.

24. Meldrum M. Toxicology of hydrogen fluoride in relation to major accident hazards. Regul Toxicol Pharmacol 1999;30:110-6.

25. Monticelli F, Toledano M, Osorio R, et al. Effect of temperature on the silane coupling agents when bonding core resin to quartz fiber posts. Dent Mater 2006; 22: 1024-8.

26. Mundim FM, Garcia Lda F, Cruvinel DR, et al. Color stability, opacity and degree of conversion of pre-heated composites. J Dent 2011; 39: e25-9.

27. Nagayassu MP, Shintome LK, Uemura ES, et al. Effect of surface treatment on the shear bond strength of a resin-based cement to porcelain. Braz Dent J 2006; 17: 290-5.

28. Newburg R, Pameijer CH. Composite resins bonded to porcelain with silane solution. J Am Dent Assoc 1978; 96 : 288-91. 
29. Oh WS, Shen C, Alegre B, et al. Wetting characteristic of ceramic to water and adhesive resin. J Prosthet Dent 2002; 88: 616-21.

30. Osorio R, Castillo-de Oyagüe R, Monticelli F, et al. Resistance to bond degradation between dualcure resin cements and pre-treated sintered CAD-CAM dental ceramics. Med Oral Patol Oral Cir Bucal 2012; 17: e669-77.

31. Ozcan M, Allahbeickaraghi A, Dündar M. Possible hazardous effects of hydrofluoric acid and recommendations for treatment approach: a review. Clin Oral Investig 2012; 16: 15-23.

32. Papacchini F, Monticelli F, Hasa I, et al. Effect of air-drying temperature on the effectiveness of silane primers and coupling blends in the repair of a microhybrid resin composite. J Adhes Dent. 2007; 9: 391-7.

33. Pashley DH, Carvalho RM, Sano H, et al. The microtensile bond test: a review. J Adhes Dent 1999; 1: 299-309.

34. Perdigão J, Gomes G, Gondo R, et al. In vitro bonding performance of all-in-one adhesives. Part I-microtensile bond strengths. J Adhes Dent. 2006; 8: 367-73.

35. Peumans M, Hikita K, De Munck J, et al. Bond durability of composite luting agents to ceramic when exposed to long-term thermocycling. Oper Dent 2007; 32: 372-9.

36. Pratt RC, Burgess JO, Schwartz RS, et al. Evaluation of bond strength of six porcelain repair systems. J Prosthet Dent 1989; 62: 11-3.

37. Queiroz JR, Benetti P, Ozcan M, et al. Surface characterization of feldspathic ceramic using ATR FT-IR and ellipsometry after various silanization protocols. Dent Mater. 2012; 28(2): 189-96.

38. Roulet JF, Söderholm KJ, Longmate J. Effects of treatment and storage conditions on ceramic/composite bond strength. J Dent Res 1995; 74: 381-7.

39. Sadek FT, Cury AH, Monticelli F, et al. The influence of the cutting speed on bond strength and integrity of microtensile specimens. Dent Mater 2005; 21: 1144-9.

40. Sano H, Shono T, Sonoda H, et al. Relationship between surface area for adhesion and tensile bond strength--evaluation of a micro-tensile bond test. Dent Mater 1994; 10: 236-40.

41. Schrader ME, Radioisotopic studies of bonding at the interface. J Adhes 1970; 2: 202-12. (abstract)

42. Shen C, Oh WS, Williams JR. Effect of post-silanization drying on the bond strength of composite to ceramic. J Prosthet Dent 2004; 91: 453-8.

43. Sorensen JA, Engelman MJ, Torres TJ, et al. Shear bond strength of composite resin to porcelain. Int J Prosthodont 1991; 4: 17-23.

44. Spohr AM, Sobrinho LC, Consani S, et al. Influence of surface conditions and silane agent on the bond of resin to IPS Empress 2 ceramic. Int J Prosthodont 2003; 16: 277-82.

45. Stangel I, Nathanson D, Hsu CS. Shear strength of the composite bond to etched porcelain. J Dent Res. 1987; 66: 1460-5.

46. Wagner WC, Aksu MN, Neme AM, et al. Effect of pre-heating resin composite on restoration microleakage. Oper Dent 2008; 33: 72-8.

47. www.gelest.com Novembro, 2011 às 14:00h.

48. Yen TW, Blackman RB, Baez RJ. Effect of acid etching on the flexural strength of a feldspathic porcelain and a castable glass ceramic. J Prosthet Dent 1993; 70: 224-33. 